\title{
The Transversal Relative Equilibria of a Hamiltonian System with Symmetry
}

\author{
G. W. Patrick \\ Department of Mathematics and Statistics \\ University of Saskatchewan \\ Saskatoon, Saskatchewan, S7N 5E6 \\ Canada \\ R. M. Roberts \\ Mathematics Institute \\ University of Warwick \\ Coventry, CV4 7AL \\ United Kingdom
}

2 February 1999

\begin{abstract}
We show that, given a certain transversality condition, the set of relative equilibria $\mathcal{E}$ near $p_{e} \in \mathcal{E}$ of a Hamiltonian system with symmetry is locally Whitney-stratified by the conjugacy classes of the isotropy subgroups (under the product of the coadjoint and adjoint actions) of the momentum-generator pairs $(\mu, \xi)$ of the relative equilibria. The dimension of the stratum of the conjugacy class $(K)$ is $\operatorname{dim} G+2 \operatorname{dim} Z(K)-\operatorname{dim} K$, where $Z(K)$ is the center of $K$, and transverse to this stratum $\mathcal{E}$ is locally diffeomorphic to the commuting pairs of the Lie algebra of $K / Z(K)$. The stratum $\mathcal{E}_{(K)}$ is a symplectic submanifold of $P$ near $p_{e} \in \mathcal{E}$ if and only if $p_{e}$ is nondegenerate and $K$ is a maximal torus of $G$. We also show that there is a dense subset of $G$-invariant Hamiltonians on $P$ for which all the relative equilibria are transversal. Thus, generically, the types of singularities that can be found in the set of relative equilibria of a Hamiltonian system with symmetry are those types found amongst the singularities at zero of the sets of commuting pairs of certain Lie subalgebras of the symmetry group.
\end{abstract}

\section{Introduction}

Let $G$ be a connected, compact Lie group, with Lie algebra $\mathfrak{g}$. The group $G$ acts via its adjoint representation on $\mathfrak{g}$ and by the dual coadjoint representation on the dual $\mathfrak{g}^{*}$ of $\mathfrak{g}$. Let $G$ act freely and symplectically on a symplectic phase 
space $(P, \omega)$ endowed with an equivariant momentum mapping $J: P \rightarrow \mathfrak{g}^{*}$. Since $G$ is acting freely, every point in $P$ is a regular point of $J$. Fix a $G$ invariant Hamiltonian $H$ on $P$. A relative equilibrium of $H$ with generator $\xi_{e} \in \mathfrak{g}$ is a point $p_{e} \in P$ for which the evolution under the flow generated by $H$ is $t \mapsto \exp \left(t \xi_{e}\right) \cdot p_{e}$. Let $G_{\mu_{e}}$ and $G_{\xi_{e}}$ respectively denote the isotropy subgroups of $\mu_{e}=J\left(p_{e}\right) \in \mathfrak{g}^{*}$ and $\xi_{e} \in \mathfrak{g}$ under the coadjoint and adjoint actions of $G$. Patrick 1995 has proved that if $G_{\mu_{e}} \cap G_{\xi_{e}}$ is a maximal torus of $G$ and a nondegeneracy condition holds then the set of relative equilibria near $p_{e}$ is a manifold of $\operatorname{dimension} \operatorname{dim} G+\operatorname{rank} G$. This manifold is symplectic under an additional nonresonance condition and the condition that $\mu_{e}$ is generic.

Recently Ortega and Ratiu [1997 have applied these results, combined with singular reduction techniques, to obtain manifolds of relative equilibria even when $p_{e}$ is not regular. In an interesting article with a number of new ideas, Montald 1997 proves that, regardless of whether or not $G$ acts freely, certain extremal relative equilibria must persist to relative equilibria on symplectic reduced spaces near to the one through $p_{e}$, and exactly counts and classifies the relative equilibria near $p_{e}$ when $G$ does act freely and the generator $\xi_{e}$ is regular. Other results on the existence of manifolds of relative equilibria through a given non-regular relative equilibrium have been obtained by Roberts and Sousa Dias [1997 and Lerman and Singer 11998.

We define the type of a pair $(\xi, \mu) \in \mathfrak{g}^{*} \oplus \mathfrak{g}$ to be the conjugacy class in $G$ of $G_{\mu} \cap G_{\xi}$ and the type of a relative equilibrium $p_{e}$ to be the type of its momentum-generator pair $\left(\mu_{e}, \xi_{e}\right)$. Generally we denote the conjugacy class of a subgroup $K \subset G$ by $(K)$, and so we say that the type of $p_{e}$ is $\left(K_{e}\right)$, where $K_{e}=G_{\mu_{e}} \cap G_{\xi_{e}}$. The main result of this paper is a description of the complete set of relative equilibria near $p_{e}$ in the relaxed situation where the type of $p_{e}$ is not necessarily a maximal torus of $G$, but where $p_{e}$ is still regular. One of our opinions, indicated first by Patrick 1995, and born out by our work, is that the momentum-generator pair is a more important object than either the momentum or generator taken singly.

Consider the case of a single 'generic' rigid body with distinct principle moments of inertia $I_{1}, I_{2}$ and $I_{3}$. This is an $S O(3)$-invariant system. The relative equilibria consist of steady rotations about the three principle axes of inertia. After the $S O(3)$ symmetry is removed by reduction to the Euler equations, the set of relative equilibria is the union of the three coordinate axes of $\mathbb{R}^{3}$. The origin has type $S O(3)$ and is the sole bifurcation point of the set of relative equilibria. The remaining equilibria have type $(S O(2))$, the 'smallest' possible. On the evidence of this example, one might expect general $S O(3)$-invariant systems to have a zero dimensional set of relative equilibria of type $S O(3)$ from which bifurcate three distinct branches of relative equilibria of type $(S O(2))$. However, the results we obtain imply this example is a poor guide to the general theory because it is nongeneric. Generically, in the class of Hamiltonian systems with symmetry group $S O(3)$, there are no regular relative equilibria of type $S O(3)$. In particular, this implies that there are no regular equilibria.

More generally let $\mathcal{E} \subset P$ denote the set of relative equilibria of a generic $G$ - 
invariant Hamiltonian system on $P$ and let $\mathcal{E} / G \subset P / G$ denote its quotient by the action of $G$. Then we show that both $\mathcal{E}$ and $\mathcal{E} / G$ are stratified by the types of the relative equilibria and that the quotient of a non-empty stratum consisting of relative equilibria of type $(K)$ has dimension equal to $2 \operatorname{dim} Z(K)-\operatorname{dim} K$, where $Z(K)$ is the center of $K$. Moreover transverse to this stratum the set $\mathcal{E} / G$ is locally isomorphic to the set of commuting pairs of the Lie algebra of $K / Z(K)$. See Theorems 11 and 2 for precise statements.

The types of transversal relative equilibria are constrained by the condition $2 \operatorname{dim} Z(K) \geq \operatorname{dim} K$, a condition which fails for $K=S O(3)$ in the rigid body example. For another example consider a generic rigid body with three rotors aligned with its (three orthogonal) principle axes. This has symmetry group $S O(3) \times S O(2)^{3}$. There is, up to symmetry, exactly one relative equilibrium of type $S O(3) \times S O(2)^{3}$, namely the equilibrium where neither the body nor the rotors move. At this equilibrium the set of relative equilibria has a singularity, bifurcating from which are very many relative equilibria of

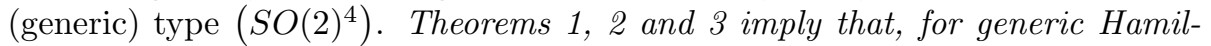
tonian systems with symmetry group $S O(3) \times S O(2)^{3}$, the relative equilibria of type $S O(3) \times S O(2)^{3}$ form isolated group orbits which are singularities of $\mathcal{E} / G$ and that near each of these singularities $\mathcal{E} / G$ is diffeomorphic to the set of commuting pairs of the Lie algebra so(3).

Here is a summary of this work: Let $\left(\mathfrak{g}^{*} \oplus \mathfrak{g}\right)^{c} \subseteq \mathfrak{g}^{*} \oplus \mathfrak{g}$ be the set of pairs $(\mu, \xi)$ such that $\operatorname{coad}_{\xi} \mu=0$, where 'coad' denotes the coadjoint representation of $\mathfrak{g}$ on $\mathfrak{g}^{*}$. Any $G$-invariant inner product on $\mathfrak{g}$ defines an isomorphism between $\mathfrak{g}$ and $\mathfrak{g}^{*}$ and between the set of commuting pairs of $\mathfrak{g}$ and the subset $\left(\mathfrak{g}^{*} \oplus \mathfrak{g}\right)^{c}$. In Section 11 we describe a stratification of $\left(\mathfrak{g}^{*} \oplus \mathfrak{g}\right)^{c}$ and in Section 2 we use this to construct stratifications of certain subsets $\mathcal{T}^{c}$ of the tangent bundle and $\mathcal{K}^{o c}$ of the cotangent bundle of $P$. A relative equilibrium is defined to be transversal if at that point the vector field is transversal to the stratification of $\mathcal{T}^{c}$ or, equivalently, the derivative of the Hamiltonian is transverse to the stratification of $\mathcal{K}^{o c}$. Some more or less standard results from transversality and stratification theory are then used to deduce Theorems 1, 2 and 3 . In Section 3 we describe a normal form for the linearization of a vector field at a transversal relative equilibrium and use this to show that near a transversal relative equilibrium $p_{e}$ the submanifold of $P$ consisting of relative equilibria of type $\left(K_{e}\right)$ is symplectic if and only if $p_{e}$ is nondegenerate and $K_{e}$ is a maximal torus in $G$ (Theorem E). This is a generalization and partial converse of a result of Patrick 1995.

\section{The Stratified Structure of $\left(\mathfrak{g}^{*} \oplus \mathfrak{g}\right)^{c}$}

The momentum-generator pair $\left(\mu_{e}, \xi_{e}\right) \in \mathfrak{g}^{*} \oplus \mathfrak{g}$ of a relative equilibrium of a $G$-invariant Hamiltonian system satisfies the relationship $\operatorname{coad}_{\xi_{e}} \mu_{e}=0$. In this section we describe the structure of the set of all pairs satisfying this relationship:

$$
\left(\mathfrak{g}^{*} \oplus \mathfrak{g}\right)^{c}=\left\{(\mu, \xi) \in \mathfrak{g}^{*} \oplus \mathfrak{g}: \operatorname{coad}_{\xi} \mu=0\right\} .
$$

Note that $\left(\mathfrak{g}^{*} \oplus \mathfrak{g}\right)^{c}$ is a $G$-invariant subvariety of $\mathfrak{g}^{*} \oplus \mathfrak{g}$ under the product 
of the coadjoint and adjoint actions. For any $(\mu, \xi) \in \mathfrak{g}^{*} \oplus \mathfrak{g}$ let $G_{\mu, \xi}$ denote the isotropy subgroup of $(\mu, \xi)$ for the product of the coadjoint and adjoint actions. Clearly $G_{\mu, \xi}=G_{\mu} \cap G_{\xi}$ where $G_{\mu}$ is the isotropy subgroup of $\mu$ for the coadjoint action of $G$ and $G_{\xi}$ is the isotropy subgroup of $\xi$ for the adjoint action. Let $\mathfrak{g}_{\mu}$ and $\mathfrak{g}_{\xi}$ denote the Lie algebras of $G_{\mu}$ and $G_{\xi}$, respectively, and $\mathfrak{g}_{\mu}^{*}$ and $\mathfrak{g}_{\xi}^{*}$ their dual spaces.

\section{Lemma 1}

1. $(\mu, \xi) \in\left(\mathfrak{g}^{*} \oplus \mathfrak{g}\right)^{c}$ if and only if $\xi \in \mathfrak{g}_{\mu} \subset \mathfrak{g}$, and $G_{\mu, \xi}=\left(G_{\mu}\right)_{\xi}$, the isotropy subgroup of $\xi$ for the adjoint action of $G_{\mu}$ on $\mathfrak{g}_{\mu}$.

2. A point $(\mu, \xi) \in \mathfrak{g}^{*} \oplus \mathfrak{g}$ lies in $\left(\mathfrak{g}^{*} \oplus \mathfrak{g}\right)^{c}$ if and only if $G_{\mu, \xi}$ contains a maximal torus of $G$.

3. If $(\mu, \xi) \in\left(g^{*} \oplus \mathfrak{g}\right)^{c}$ then $\mathfrak{g}_{\mu, \xi}=\mathfrak{g}_{\mu} \cap \mathfrak{g}_{\xi}$ is abelian if and only if $G_{\mu, \xi}$ is a maximal torus of $G$.

Proof We have

$$
(\mu, \xi) \in\left(\mathfrak{g}^{*} \oplus \mathfrak{g}\right)^{c} \Leftrightarrow \operatorname{coad}_{\xi} \mu=0 \Leftrightarrow \xi \in \mathfrak{g}_{\mu}
$$

Moreover

$$
G_{\mu, \xi}=G_{\mu} \cap G_{\xi}=\left\{g \in G_{\mu}: \operatorname{Ad}_{g} \xi=\xi\right\}
$$

and part 1 follows from the fact that the adjoint action of $G$ on $\mathfrak{g}$ restricts to the adjoint action of $G_{\mu}$ on $\mathfrak{g}_{\mu}$.

For part 2, suppose first that $(\mu, \xi) \in\left(\mathfrak{g}^{*} \oplus \mathfrak{g}\right)^{c}$. Then, by part $1, G_{\mu, \xi}$ is an isotropy subgroup of the adjoint action of $G_{\mu}$ and so contains a maximal torus of $G_{\mu}$. However $G_{\mu}$ is an isotropy subgroup of the coadjoint action of $G$ and so maximal tori of $G_{\mu}$ are also maximal tori of $G$.

Conversely, suppose $G_{\mu, \xi}$ contains a maximal torus $T$ of $G$. Then $(\mu, \xi) \in$ $\operatorname{fix}\left(T ; \mathfrak{g}^{*} \oplus \mathfrak{g}\right)=\mathfrak{t}^{*} \oplus \mathfrak{t}$ where $\mathfrak{t}$ is the Lie algebra of $T$. Since $T$ is abelian it follows that $\operatorname{coad}_{\xi} \mu=0$ and so $(\mu, \xi) \in\left(\mathfrak{g}^{*} \oplus \mathfrak{g}\right)^{c}$.

For part 3 , if $G_{\mu, \xi}$ is a maximal torus then its Lie algebra $\mathfrak{g}_{\mu, \xi}$ is abelian. Conversely, suppose $\mathfrak{g}_{\mu, \xi}$ is abelian. Then by Proposition 4.25 of Adams 1969. and by part $1, G_{\mu, \xi}$ is connected and so is also abelian. Thus $G_{\mu, \xi}$ is a torus that contains a maximal torus, and so is a maximal torus.

For any set $X$ with an action of $G$ we denote the subset consisting of points with isotropy subgroup conjugate to $K \subset G$ by $X_{(K)}$. We will denote the set of conjugacy classes of isotropy subgroups of the action of $G$ on $\left(\mathfrak{g}^{*} \oplus \mathfrak{g}\right)^{c}$ by $\mathcal{I}^{c}$. By Lemma 1, $\mathcal{I}^{c}$ is the set of conjugacy classes of isotropy subgroups of the action of $G$ on $\mathfrak{g}^{*} \oplus \mathfrak{g}$ which contain a maximal torus of $G$, and

$$
\left(\mathfrak{g}^{*} \oplus \mathfrak{g}\right)^{c}=\bigsqcup_{(K) \in \mathcal{I}^{c}}\left(\mathfrak{g}^{*} \oplus \mathfrak{g}\right)_{(K)}
$$


By the general theory of actions of a compact group, each set $\left(\mathfrak{g}^{*} \oplus \mathfrak{g}\right)_{(K)}$ is a manifold and the collection of these manifolds is a Whitney regular stratification of $\mathfrak{g}^{*} \oplus \mathfrak{g}$. Since $\left(\mathfrak{g}^{*} \oplus \mathfrak{g}\right)^{c}$ is the union of all the strata of $\mathfrak{g}^{*} \oplus \mathfrak{g}$ which are smaller than the stratum of the maximal tori, it follows that $\left\{\left(\mathfrak{g}^{*} \oplus \mathfrak{g}\right)_{(K)}:(K) \in \mathcal{I}^{c}\right\}$ is a Whitney regular stratification of $\left(\mathfrak{g}^{*} \oplus \mathfrak{g}\right)^{c}$. The general theory also tells us that the quotients of the strata by the action of $G$ are smooth manifolds and that the set of these strata is a Whitney regular stratification of the orbit space $\left(\mathfrak{g}^{*} \oplus \mathfrak{g}\right) / G$. It follows that the set of quotients of the strata $\left(\mathfrak{g}^{*} \oplus \mathfrak{g}\right)_{(K)}$ with $(K) \in \mathcal{I}^{c}$ is a Whitney regular stratification of $\left(\mathfrak{g}^{*} \oplus \mathfrak{g}\right)^{c} / G$.

The next proposition describes the local structure of these stratifications. We fix a $G$-invariant inner product on $\mathfrak{g}$, which determines a $G$-equivariant isomorphism between $\mathfrak{g}$ and $\mathfrak{g}^{*}$ and hence also a $G$-invariant inner product on $\mathfrak{g}^{*}$. The $G$-invariant inner product also defines an isomorphism between $\left(\mathfrak{g}^{*} \oplus \mathfrak{g}\right)^{c}$ and the set of commuting pairs of $\mathfrak{g}$ :

$$
(\mathfrak{g} \oplus \mathfrak{g})^{c}=\left\{(\eta, \xi) \in \mathfrak{g} \oplus \mathfrak{g}: \operatorname{ad}_{\xi} \eta=[\xi, \eta]=0\right\} .
$$

If $\mathfrak{k}$ is a subspace of $\mathfrak{g}$ the inner product on $\mathfrak{g}^{*}$ is used to identify $\mathfrak{k}^{*}$ with the subspace ann $(\mathfrak{k})^{\perp}$ of $\mathfrak{g}^{*}$. Here ann $(\mathfrak{k})$ is the annihilator of $\mathfrak{k}$ in $\mathfrak{g}^{*}$ and ' $\perp$ ' denotes the orthogonal complement with respect to the $G$-invariant inner product on $\mathfrak{g}^{*}$.

Suppose $(\mu, \xi) \in\left(\mathfrak{g}^{*} \oplus \mathfrak{g}\right)^{c}$ and let $K=G_{\mu} \cap G_{\xi}$. Denote the Lie algebra of $K$ by k. Let $\chi: U \rightarrow G$ be a section of the natural projection $G \rightarrow G / K$, defined on an open neighborhood $U$ of $K$ in $G / K$ and such that $\chi(K)$ is the identity in $G$. Give $\mathfrak{g}^{*} \oplus \mathfrak{g}$ and $\left(\mathfrak{g}^{*} \oplus \mathfrak{g}\right)^{c}$ the stratifications obtained by taking the connected components of the orbit type strata for the actions of $G$. Similarly give $\mathfrak{k}^{*} \oplus \mathfrak{k}$ and $\left(\mathfrak{k}^{*} \oplus \mathfrak{k}\right)^{c}$ the corresponding stratifications obtained from the actions of $K$. Extend these stratifications to $U \times\left(\mathfrak{k}^{*} \oplus \mathfrak{k}\right)$ and $U \times\left(\mathfrak{k}^{*} \oplus \mathfrak{k}\right)^{c}$ by taking the product of each stratum with $U$. We will always consider any open subset of a stratified set to be automatically endowed with the stratification obtained by taking the intersections of the strata with the open subset.

Proposition 1 Under the above assumptions, there exists a $G$-invariant open neighborhood $W$ of $(\mu, \xi)$ in $\mathfrak{g}^{*} \oplus \mathfrak{g}$ and a $K$-invariant open neighborhood $V$ of the origin in $\mathfrak{k}^{*} \oplus \mathfrak{k}$ such that the map

$$
\begin{aligned}
& \Sigma: U \times V \rightarrow \mathfrak{g}^{*} \oplus \mathfrak{g} \\
& (u,(\nu, \eta)) \mapsto \chi(u) \cdot((\mu, \xi)+(\nu, \eta))
\end{aligned}
$$

is an embedding of $U \times V$ into $W$ which restricts to an isomorphism of smoothly stratified spaces between $U \times\left(V \cap\left(\mathfrak{k}^{*} \oplus \mathfrak{k}\right)^{c}\right)$ and $W \cap\left(\mathfrak{g}^{*} \oplus \mathfrak{g}\right)^{c}$.

Proof By the theory of compact group actions there is a $K$-invariant neighborhood $W$ of $(\mu, \xi)$ in $\mathfrak{g}^{*} \oplus \mathfrak{g}$ such that

$$
S_{\mu, \xi}=W \cap\left((\mu, \xi)+(\mathfrak{g} \cdot(\mu, \xi))^{\perp}\right)
$$


is a $K$-invariant slice to the action of $G$ on $\mathfrak{g}^{*} \oplus \mathfrak{g}$ at $(\mu, \xi)$. A straightforward calculation shows that $\mathfrak{k}^{*} \oplus \mathfrak{k}$ is contained in $(\mathfrak{g} \cdot(\mu, \xi))^{\perp}$. Let $V$ be the intersection of $\mathfrak{k}^{*} \oplus \mathfrak{k}$ with $S_{\mu, \xi}-(\mu, \xi)$. Then it follows from the slice theorem that $\Sigma$ is an embedding of $U \times V$ into $W$.

It remains to be proved that

$$
S_{\mu, \xi} \cap\left(\mathfrak{g}^{*} \oplus \mathfrak{g}\right)^{c}=(\mu, \xi)+V \cap\left(\mathfrak{k}^{*} \oplus \mathfrak{k}\right)^{c} .
$$

The righthand side of this equation is clearly contained in the lefthand side, so it is sufficient to prove that the lefthand side is contained in the righthand side. Note that for any $(\nu, \eta) \in\left(\mathfrak{g}^{*} \oplus \mathfrak{g}\right)$ we always have $\nu \in \mathfrak{g}_{\nu}^{*}$ and $\eta \in \mathfrak{g}_{\eta}$. If $(\nu, \eta) \in\left(\mathfrak{g}^{*} \oplus \mathfrak{g}\right)^{c}$ then we also have $\nu \in \mathfrak{g}_{\eta}^{*}$ and $\eta \in \mathfrak{g}_{\nu}$, and hence $\nu \in \mathfrak{g}_{\eta}^{*} \cap \mathfrak{g}_{\nu}^{*}$ and $\eta \in \mathfrak{g}_{\eta} \cap \mathfrak{g}_{\nu}$. If $(\nu, \eta)$ lies in the slice $S_{\mu, \xi}$ then the isotropy subgroup $G_{\nu, \eta}$ must be contained in $K$, and so $\mathfrak{g}_{\eta} \cap \mathfrak{g}_{\nu} \subset \mathfrak{k}$. It follows that if $(\nu, \eta) \in S_{\mu, \xi} \cap\left(\mathfrak{g}^{*} \oplus \mathfrak{g}\right)^{c}$ then $(\nu, \eta)$ must lie in $\mathfrak{k}^{*} \oplus \mathfrak{k}$, and hence in $\left(\mathfrak{k}^{*} \oplus \mathfrak{k}\right)^{c}$, as required.

This proposition shows that the local structure of the stratification of $\left(\mathfrak{g}^{*} \oplus\right.$ $\mathfrak{g})^{c}$ near a point with isotropy subgroup $K$ can be reduced to that of the set $\left(\mathfrak{k}^{*} \oplus \mathfrak{k}\right)^{c}$. For any $(K) \in \mathcal{I}^{c}$ let $\mathcal{I}^{c}(K)$ denote the subset of $\mathcal{I}^{c}$ consisting of orbit types $\left(K^{\prime}\right)$ for which the closure of the stratum $\left(\mathfrak{g}^{*} \oplus \mathfrak{g}\right)_{\left(K^{\prime}\right)}$ contains $\left(\mathfrak{g}^{*} \oplus \mathfrak{g}\right)_{(K)}$. Then the proof of the Proposition implies that $\mathcal{I}^{c}(K)$ is the set of conjugacy classes in $G$ of the isotropy subgroups of the action of $K$ on $\left(\mathfrak{k}^{*} \oplus \mathfrak{k}\right)^{c}$.

The next result, which is valid for any connected compact Lie group $K$, gives a further reduction. Let $Z(K)$ denote the center of $K$. Denote the quotient $K / Z(K)$ by $L$, its Lie algebra by $\mathfrak{l}$ and the Lie algebra of $Z(K)$ by $\mathfrak{z}$. Identify $\mathfrak{z}^{*}$ with the subspace $\operatorname{ann}(\mathfrak{z})^{\perp}$ of $\mathfrak{k}^{*}$.

Proposition $2 A K$-invariant inner product on $\mathfrak{k}$ determines a $K$-equivariant linear isomorphism

$$
\sigma: \mathfrak{k}^{*} \oplus \mathfrak{k} \cong\left(\mathfrak{l}^{*} \oplus \mathfrak{l}\right) \oplus\left(\mathfrak{z}^{*} \oplus \mathfrak{z}\right)
$$

where the action of $K$ on $\mathfrak{k}^{*} \oplus \mathfrak{k}$ is the product of coadjoint and adjoint actions, the action on $\mathfrak{l}^{*} \oplus \mathfrak{l}$ factors through the product of the coadjoint and adjoint actions of $L$ and the action on $\mathfrak{z}^{*} \oplus \mathfrak{z}$ is trivial. Moreover $\sigma$ maps $\operatorname{fix}\left(K ; \mathfrak{k}^{*} \oplus \mathfrak{k}\right)$ isomorphically to $\mathfrak{z}^{*} \oplus \mathfrak{z}$ and $\left(\mathfrak{k}^{*} \oplus \mathfrak{k}\right)^{c}$ to $\left(\mathfrak{l}^{*} \oplus \mathfrak{l}\right)^{c} \oplus\left(\mathfrak{z}^{*} \oplus \mathfrak{z}\right)$.

Proof Since $K$ acts by the adjoint action on $\mathfrak{k}, \mathfrak{z}=\operatorname{fix}(K ; \mathfrak{k})$. The natural projection from $\mathfrak{k}$ to $\mathfrak{k} / \mathfrak{z} \cong \mathfrak{l}$ is equivariant with respect to the natural actions of $K$ on $\mathfrak{k}$ and $\mathfrak{l}$ and so induces an isomorphism of representations between $\operatorname{fix}(K ; \mathfrak{k})^{\perp}$ and $\mathfrak{l}$. Hence $\mathfrak{k}$ is isomorphic as a representation of $K$ to $\mathfrak{l} \oplus \mathfrak{z}$. The invariant inner product translates this into an isomorphism $\mathfrak{k}^{*} \cong \mathfrak{l}^{*} \oplus \mathfrak{z}^{*}$ and putting the two together gives $\sigma$. Since the adjoint action of $\mathfrak{z}$ on $\mathfrak{k}$ is trivial $\sigma$ $\operatorname{maps}\left(\mathfrak{k}^{*} \oplus \mathfrak{k}\right)^{c}$ to $\left(\mathfrak{l}^{*} \oplus \mathfrak{l}\right)^{c} \oplus\left(\mathfrak{z}^{*} \oplus \mathfrak{z}\right)$. 
Thus the orbit type stratification of $\left(\mathfrak{k}^{*} \oplus \mathfrak{k}\right)^{c}$ is isomorphic to the stratification of $\left(\mathfrak{l}^{*} \oplus \mathfrak{l}\right)^{c} \oplus\left(\mathfrak{z}^{*} \oplus \mathfrak{z}\right)$ obtained by taking the products of the orbit type strata of $\left(\mathfrak{l}^{*} \oplus \mathfrak{l}\right)^{c}$ with $\left(\mathfrak{z}^{*} \oplus \mathfrak{z}\right)$.

Corollary 1 If $(K) \in \mathcal{I}^{c}$ then the dimension of $\left(\mathfrak{g}^{*} \oplus \mathfrak{g}\right)_{(K)}^{c}$ is equal to $\operatorname{dim} G+$ $2 \operatorname{dim} Z(K)-\operatorname{dim} K$.

Proof By Proposition 1

$$
\operatorname{dim}\left(\mathfrak{g}^{*} \oplus \mathfrak{g}\right)_{(K)}^{c}=\operatorname{dim} U+\operatorname{dim}\left(\mathfrak{k}^{*} \oplus \mathfrak{k}\right)_{(K)}^{c} .
$$

The dimension of $U$ is $\operatorname{dim} G-\operatorname{dim} K$ while by Proposition 2 the dimension of

$$
\left(\mathfrak{k}^{*} \oplus \mathfrak{k}\right)_{(K)}^{c}=\operatorname{fix}\left(K ; \mathfrak{k}^{*} \oplus \mathfrak{k}\right)=\mathfrak{z}^{*} \oplus \mathfrak{z}
$$

is $2 \operatorname{dim} \mathfrak{z}$. This gives the result.

We note that these structure results also follow from the results of Arms, Marsden, and Moncrief 1981 and Sjamaar and Lerman 1991 on the structure of level sets of momentum maps. Indeed, the product of the coadjoint and adjoint actions of $G$ on $\mathfrak{g}^{*} \oplus \mathfrak{g}$ is symplectic with respect to the natural 'cotangent bundle' symplectic structure, the map $(\nu, \eta) \mapsto \operatorname{coad}_{\eta} \nu$ is an equivariant momentum map for this action, and $\left(\mathfrak{g}^{*} \oplus \mathfrak{g}\right)^{c}$ is its zero level set. Moreover the symplectic normal space to the group orbit through any point $(\mu, \xi) \in\left(\mathfrak{g}^{*} \oplus \mathfrak{g}\right)^{c}$ with isotropy subgroup $K$ can be identified with $\mathfrak{k}^{*} \oplus \mathfrak{k}$. This approach also shows that the strata $\left(\mathfrak{g}^{*} \oplus \mathfrak{g}\right)_{(K)}^{c}$ are symplectic submanifolds of $\mathfrak{g}^{*} \oplus \mathfrak{g}$.

\section{Transversal Relative Equilibria}

In this section we first define what we mean by a transversal relative equilibrium (Definition 1) and then give the main results on the structure of the space of relative equilibria near a transversal relative equilibrium (Theorems 1 1 and 2) and on the genericity of the set of Hamiltonians for which all the relative equilibria are transversal (Theorem 3).

Define the map $\widetilde{J}: P \times \mathfrak{g} \rightarrow \mathfrak{g}^{*} \oplus \mathfrak{g}$ by $(p, \xi) \mapsto(J(p), \xi)$. Since $G$ is acting freely the map $J$ is a $G$-equivariant submersion and hence so also is $\widetilde{J}$. Define

$$
(P \times \mathfrak{g})^{c}=\widetilde{J}^{-1}\left(\mathfrak{g}^{*} \oplus \mathfrak{g}\right)^{c}=\left\{(p, \xi) \in P \times \mathfrak{g}: \operatorname{coad}_{\xi} J(p)=0\right\} .
$$

If $p_{e}$ is a relative equilibrium of a $G$-invariant Hamiltonian system on $P$ with generator $\xi_{e}$ then $\left(p_{e}, \xi_{e}\right) \in(P \times \mathfrak{g})^{c}$. The fact that $\widetilde{J}$ is a submersion implies that the Whitney regular stratification of $\left(\mathfrak{g}^{*} \oplus \mathfrak{g}\right)^{c}$ pulls back to a Whitney regular stratification of $(P \times \mathfrak{g})^{c}$. The strata of $(P \times \mathfrak{g})^{c}$ are the nonempty submanifolds

$$
\begin{aligned}
(P \times \mathfrak{g})_{(K)} & =\widetilde{J}^{-1}\left(\left(\mathfrak{g}^{*} \oplus \mathfrak{g}\right)_{(K)}\right) \\
& =\left\{(p, \xi) \in P \times \mathfrak{g}: G_{J(p), \xi} \text { is conjugate to } K\right\}
\end{aligned}
$$


where $(K) \in \mathcal{I}^{c}$.

Let $\mathcal{T}$ denote the $G$-invariant subbundle of the vector bundle $T P$ with fiber over $p \in P$ equal to the tangent space at $p$ to the orbit of $G$ through $p$, that is $\mathcal{T}_{p}=\mathfrak{g} . p$. The subbundle $\mathcal{T}$ is the image of the map $I: P \times \mathfrak{g} \rightarrow T P$ defined by $(p, \xi) \mapsto \xi . p$. Since $G$ is acting freely on $P$, the map $I$ is a $G$-equivariant vector bundle isomorphism between $P \times \mathfrak{g}$ (considered as a bundle over $P$ ) and $\mathcal{T}$.

Let $\mathcal{K}$ denote the $G$-invariant subbundle of the vector bundle $T P$ with fiber over $p \in P$ given by $\mathcal{K}_{p}=\operatorname{ker} d J(p)$. Note that the Hamiltonian vector field of any $G$-invariant Hamiltonian on $P$ takes values in $\mathcal{K}$. Let $\mathcal{T}^{c}=\mathcal{T} \cap \mathcal{K}$, a $G$-invariant subset of $\mathcal{K}$. For each $(K) \in \mathcal{I}^{c}$ define the set $\mathcal{T}_{(K)}^{c}$ by

$$
\mathcal{T}_{(K)}^{c}=\left\{\xi . p \in \mathcal{T}^{c}:(J(p), \xi) \text { has type }(K)\right\} .
$$

Let $\mathcal{T}^{o}$ and $\mathcal{K}^{o}$ denote the $G$-invariant vector subbundles of $T^{*} P$ with fibers

$$
\mathcal{T}_{p}^{o}=\operatorname{ann}\left(\mathcal{T}_{p}\right), \quad \mathcal{K}_{p}^{o}=\operatorname{ann}\left(\mathcal{K}_{p}\right) .
$$

The subbundle $\mathcal{K}^{o}$ is the image of the map $I^{o}: P \times \mathfrak{g} \rightarrow T^{*} P$ defined by $(p, \xi) \mapsto d J_{\xi}(p)$. Moreover, since $G$ is acting freely on $P$, the map $I^{o}$ is a $G$ equivariant vector bundle isomorphism between $P \times \mathfrak{g}$ (considered as a bundle over $P$ ) and $\mathcal{K}^{\circ}$. Note that any $G$-invariant Hamiltonian $P$ is such that $d H$ has values in $\mathcal{T}^{o}$. Let $\mathcal{K}^{o c}=\mathcal{T}^{o} \cap \mathcal{K}^{o}$, a $G$-invariant subset of $\mathcal{T}^{o}$. For each $(K) \in \mathcal{I}^{c}$ define the set $\mathcal{K}_{(K)}^{o c}$ by

$$
\mathcal{K}_{(K)}^{o c}=\left\{d J_{\xi}(p) \in \mathcal{K}^{o c}:(J(p), \xi) \text { has type }(K)\right\} .
$$

\section{Proposition 3}

1. The set $\left\{\mathcal{T}_{(K)}^{c}:(K) \in \mathcal{I}^{c}\right\}$ is a Whitney regular stratification of $\mathcal{T}^{c}$ and the diffeomorphism $I: P \times \mathfrak{g} \rightarrow \mathcal{T}$ restricts to a stratum preserving bijection of $(P \times \mathfrak{g})^{c}$ to $\mathcal{T}^{c}$.

2. The set $\left\{\mathcal{K}_{(K)}^{o c}:(K) \in \mathcal{I}^{c}\right\}$ is a Whitney regular stratification of $K^{\text {oc }}$ and the diffeomorphism $I^{o}:(P \times \mathfrak{g}) \rightarrow \mathcal{K}^{o}$ restricts to a stratum preserving bijection of $(P \times \mathfrak{g})^{c}$ to $\mathcal{K}^{o c}$.

Proof For part 1, equivariance of $J$ implies that $d J(p)(\xi . p)=-\operatorname{coad}_{\xi} J(p)$ and so

$$
I(p, \xi) \in \mathcal{T}^{c} \Leftrightarrow \xi . p \in \mathcal{K} \Leftrightarrow 0=-\operatorname{coad}_{\xi} J(p) \Leftrightarrow(p, \xi) \in(P \times \mathfrak{g})^{c} .
$$

Hence $I$ is a diffeomorphism which maps $(P \times \mathfrak{g})^{c}$ bijectively to $\mathcal{T}^{c}$. The strata $\mathcal{T}_{(K)}^{c}$ are the images under $I$ of the strata of $(P \times \mathfrak{g})^{c}$ and so define a Whitney regular stratification of $T^{c}$. The map $I$ is stratum preserving by construction. Part 2 is similar. 
Let $H: P \rightarrow \mathbb{R}$ be a $G$-invariant Hamiltonian function on $P, d H: P \rightarrow \mathcal{T}^{\circ} \subset$ $T^{*} P$ its derivative and $X_{H}: P \rightarrow \mathcal{K} \subset T P$ the corresponding $G$-equivariant Hamiltonian vector field. Denote by $\psi: P \times \mathfrak{g} \rightarrow T P$ the map $(p, \xi) \mapsto X_{H}(p)-$ $\xi . p$, and let $\psi_{(K)}$ denote the restriction of $\psi$ to the submanifold $(P \times \mathfrak{g})_{(K)}^{c}$ of $P \times \mathfrak{g}$. Denote by $\psi^{o}: P \times \mathfrak{g} \rightarrow T^{*} P$ the map $(p, \xi) \mapsto d H(p)-d J_{\xi}(p)$ and let $\psi_{(K)}^{o}$ denote its restriction to $(P \times \mathfrak{g})_{(K)}^{c}$. Note that $\psi_{(K)}$ maps into $\mathcal{K}$ and $\psi_{(K)}^{o}$ into $\mathcal{T}^{o}$. The following lemma is a direct consequence of the definitions.

Lemma 2 The vector field $X_{H}$ has a relative equilibrium at $p_{e}$ if and only if the following equivalent conditions hold:

1. $X_{H}\left(p_{e}\right) \in \mathcal{T}^{c}$;

2. $d H\left(p_{e}\right) \in \mathcal{K}^{o c}$;

3. There exists $\xi_{e} \in \mathfrak{g}$ such that $\left(p_{e}, \xi_{e}\right) \in(P \times \mathfrak{g})^{c}$ and $\psi\left(p_{e}, \xi_{e}\right)=0$;

4. There exists $\xi_{e} \in \mathfrak{g}$ such that $\left(p_{e}, \xi_{e}\right) \in(P \times \mathfrak{g})^{c}$ and $\psi^{o}\left(p_{e}, \xi_{e}\right)=0$.

In statements 3 and 4 the element $\xi_{e}$ is the generator of the relative equilibrium. Moreover, if $p_{e}$ is a relative equilibrium of $X_{H}$ with generator $\xi_{e}$, then

$$
\begin{aligned}
p_{e} \text { has type }(K) \Leftrightarrow\left(p_{e}, \xi_{e}\right) \in(P & \times \mathfrak{g})_{(K)}^{c} \\
& \Leftrightarrow X_{H}\left(p_{e}\right) \in \mathcal{T}_{(K)}^{c} \Leftrightarrow d H\left(p_{e}\right) \in \mathcal{K}_{(K)}^{o c} .
\end{aligned}
$$

The following lemma and the accompanying definition identify what we mean by a transversal relative equilibrium. As we shall show, transversality is generic in the class of symmetric Hamiltonian systems.

Lemma 3 Let $p_{e}$ be a relative equilibrium of $X_{H}$ with generator $\xi_{e}$ and momentum $\mu_{e}=J\left(p_{e}\right)$, and let $K_{e}=G_{\mu_{e}} \cap G_{\xi_{e}}$. Then the following are equivalent:

1. $X_{H}: P \rightarrow \mathcal{K}$ is transversal to $\mathcal{T}_{\left(K_{e}\right)}^{c}$ at $p_{e}$;

2. $d H: P \rightarrow \mathcal{T}^{o}$ is transversal to $\mathcal{K}_{\left(K_{e}\right)}^{o c}$ at $p_{e}$;

3. $\psi_{\left(K_{e}\right)}:(P \times \mathfrak{g})_{\left(K_{e}\right)}^{c} \rightarrow \mathcal{K}$ is transversal to the zero section of $\mathcal{K}$ at $\left(p_{e}, \xi_{e}\right)$;

4. $\psi_{\left(K_{e}\right)}^{o}:(P \times \mathfrak{g})_{\left(K_{e}\right)}^{c} \rightarrow \mathcal{T}^{o}$ is transversal to the zero section of $\mathcal{T}^{o}$ at $\left(p_{e}, \xi_{e}\right)$.

Proof The symplectic form $\omega$ on $P$ defines a vector bundle isomorphism $\omega^{b}$ : $T P \rightarrow T^{*} P$ which converts part 1 into part 2 , since

$$
\omega^{b} \circ X_{H}=d H, \quad \omega^{b}\left(\mathcal{K}_{e}\right)=T^{o}, \quad \omega^{b}\left(\mathcal{T}_{\left(K_{e}\right)}^{c}\right)=\mathcal{K}_{\left(K_{e}\right)}^{o c} .
$$

Thus (1) is equivalent to (2) and similarly (3) is equivalent to (4), so we need only show the equivalence of (1) and (3). 
Generally, as is easily verified, given a section $X$ of a vector bundle $\pi: E \rightarrow P$ and a mapping $f$ from a manifold $M$ to $E$ over $f_{0}: M \rightarrow P$, the condition that $X$ and $f$ are transversal is equivalent to the condition that $f-X \circ f_{0}$ is transversal to the zero section $Z(E)$ of $E$. When applied to the diagram

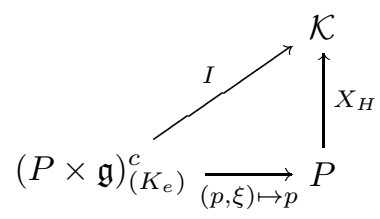

this shows that (3) is equivalent to the statement that $I \mid(P \times \mathfrak{g})_{\left(K_{e}\right)}^{c}=\mathcal{T}_{\left(K_{e}\right)}^{c}$ is transversal to $X_{H}$, and so is equivalent to (1).

Definition 1 A relative equilibrium $p_{e}$ is said to be transversal if the equivalent conditions in Lemma 3 hold.

Let $\mathcal{E} \subset P$ denote the set of all relative equilibria of $X_{H}$ and $\mathcal{E}_{(K)}$ the subset consisting of those of type $K$.

Theorem 1 Let $p_{e}$ be a relative equilibrium of $X_{H}$ with generator $\xi_{e}$ and momentum $\mu_{e}=J\left(p_{e}\right)$, and let $K_{e}=G_{\mu_{e}} \cap G_{\xi_{e}}$. If $p_{e}$ is transversal then there exists a $G$-invariant open neighborhood $U$ of $p_{e}$ in $P$ such that

1. every relative equilibrium of $X_{H}$ in $\mathcal{E} \cap U$ is transversal;

2. for each $(K) \in \mathcal{I}^{c}\left(K_{e}\right)$ the subset $\mathcal{E}_{(K)} \cap U$ is a submanifold of dimension $\operatorname{dim} G+2 \operatorname{dim} Z(K)-\operatorname{dim} K$ and its quotient by the action of $G,\left(\mathcal{E}_{(K)} \cap\right.$ $U) / G$ is a manifold of dimension $2 \operatorname{dim} Z(K)-\operatorname{dim} K$;

3. the sets $\left\{\mathcal{E}_{(K)} \cap U:(K) \in \mathcal{I}^{c}\left(K_{e}\right)\right\}$ and $\left\{\left(\mathcal{E}_{(K)} \cap U\right) / G:(K) \in \mathcal{I}^{c}\left(K_{e}\right)\right\}$ are Whitney regular stratifications of $\mathcal{E} \cap U$ and $(\mathcal{E} \cap U) / G$, respectively.

Proof Since $\mathcal{E}=X_{H}^{-1}\left(\mathcal{T}^{c}\right)$ the statements about $\mathcal{E}$ follow directly from the transversality of $X_{H}$ at $p_{e}$ to the Whitney regular stratification of $\mathcal{T}^{c}$ described above. The results on $\mathcal{E} / G$ follow from those on $\mathcal{E}$ and the fact that $G$ acts freely on $P$.

The possible types of a transversal relative equilibrium are severely restricted by the following corollary. For example, if $G$ has no center then no relative equilibria can have type $(G)$.

Corollary 2 If $p_{e}$ is a transversal relative equilibrium then

$$
\operatorname{dim} Z\left(K_{e}\right) \geq \frac{1}{2} \operatorname{dim} K_{e}
$$


Proof This is immediate since $\operatorname{dim} \mathcal{E}_{\left(K_{e}\right)} / G=2 \operatorname{dim} Z\left(K_{e}\right)-\operatorname{dim} K_{e}$ is nonnegative.

We next give a more detailed description of the local structure of the set $\mathcal{E}$. First we recall some properties of Whitney stratifications, as described in Goresky and MacPherson 1988. Let $M$ be a manifold, $Z \subset M$ a Whitney stratified space, $p \in Z$, and $S$ the stratum through $p$. Let $N^{\prime}$ be a submanifold of $M$ transverse to $S$ at $p$, meaning that $T_{p} M=T_{p} S \oplus T_{p} N^{\prime}$. Choose a Riemannian metric on $N^{\prime}$. Then for small enough $\delta$ the normal slice $N=N^{\prime} \cap Z \cap B_{\delta}(p)$ and the link $L=N^{\prime} \cap Z \cap \partial B_{\delta}(p)$ have topological types independent of $\delta$, the Riemannian metric and $N^{\prime}$. Moreover $N$ is a cone over $L$ and locally $Z$ is the product of $N$ and $S$. These result follow from the Thom isotopy lemma.

Suppose that near $p$ the set $Z$ is a smooth stratum preserving embedding of the product of a fixed stratified cone $C$ in some vector space $\mathbb{E}$ and an open subset $U$ of Euclidean space. Proposition 1 shows that this applies to points in $\left(\mathfrak{g}^{*} \oplus \mathfrak{g}\right)^{c}$. Without loss of generality we may assume that the cone linearly spans $\mathbb{E}$. Then we will say that $Z$ has (smooth) singularity-type $C$ at $p$. We make the following elementary observations, all based on more or less transparent applications of the implicit function theorem:

1. Given any transverse submanifold $N^{\prime}$, the normal space $N$ is the image of a smooth embedding of $C$ into $N^{\prime}$. Conversely, if $N$ is such for some such $N^{\prime}$ then $Z$ has singularity type $C$ at $p$.

2. Suppose $M^{\prime}$ is a submanifold of $M$ and $Z \subseteq M^{\prime}$. Take any transverse submanifold $N^{\prime \prime}$ in $M^{\prime}$ to the stratum $S$ and extend it to a transverse submanifold $N^{\prime}$ to $S$ within $M$. By Item (1) just above the normal space $N$ is the image of $C$ by a smooth embedding $\iota: \mathbb{E} \rightarrow N^{\prime}$, say. Then $\iota(\mathbb{E})$ is a submanifold with tangent space contained in $N^{\prime \prime}$, and consequently $\iota$ may be deformed so that its image is contained in $N^{\prime \prime}$, all the while fixing its values on $C$. The point of this is that $Z$ also has singularity-type $C$ at $p$ when it is regarded as a subset of $M^{\prime}$.

3. If $N$ is some manifold and $f: N \rightarrow M$ is a smooth map such that $f(n)=p$ and $f$ is transversal to the stratum $S$ at $n$, then $f^{-1}(Z)$ is a stratified space with the same singularity type at $n$ as that of $Z$ at $p$. For this we invoke smooth coordinates at $z$ such that $Z$ locally becomes $C \times S \times\{0\} \subset$ $\mathbb{E} \times S \times \mathbb{F}$, where $\mathbb{F}$ is some vector space. Then $f$ is transversal to $\mathbb{E} \times S \times\{0\}$, so we may replace $f$ by its restriction to $f^{-1}(\mathbb{E} \times S \times\{0\})$, thereby removing $\mathbb{F}$. Let $\pi_{1}: \mathbb{E} \times S \rightarrow \mathbb{E}$ be the projection. Again by transversality, $\pi_{1} \circ f$ is a submersion at $n$ and so, by local diffeomorphism of $N$ near $n, \pi_{1} \circ f$ becomes the projection $(x, y) \mapsto x$, and consequently $f^{-1}(Z)$ also has singularity-type $C$ at $n$.

Our point is just that these singularity theory results are obtainable in the smooth category without the use of the Thom isotopy lemma provided the smooth structure of the stratified spaces is apriori known, as in Proposition 1 . 
Theorem 2 The stratified spaces $\mathcal{E} \cap U$ and $(\mathcal{E} \cap U) / G$ of Theorem $\mathbb{1}$ both have singularity type $\left(\mathfrak{l}_{e}^{*} \oplus \mathfrak{l}_{e}\right)^{c}$ at $p_{e}$ and $\bar{p}_{e}=G$. $p_{e}$, respectively, where $\mathfrak{l}_{e}$ is the Lie algebra of $K_{e} / Z\left(K_{e}\right)$.

Proof By Propositions 1 and 2, the set $(\mathfrak{g} \oplus \mathfrak{g})^{c}$ has singularity type $\left(\mathfrak{l}_{e}^{*} \oplus \mathfrak{l}_{e}\right)^{c}$ at $\left(J\left(p_{e}\right), \xi_{e}\right)$. Since $J \times \operatorname{Id}: P \times \mathfrak{g} \rightarrow \mathfrak{g}^{*} \times \mathfrak{g}$ is a submersion, the singularity type of $(P \times \mathfrak{g})^{c}=(J \times \mathrm{Id})^{-1}\left(\mathfrak{g}^{*} \oplus \mathfrak{g}\right)^{c}$ at $\left(p_{e}, \xi_{e}\right)$ is also $\left(\mathfrak{l}_{e}^{*} \oplus \mathfrak{l}_{e}\right)^{c}$. The set $T^{c}$ also has this singularity type at the point $\left(p_{e}, \xi_{e} \cdot p_{e}\right)$, since it is mapped diffeomorphically to $(P \times \mathfrak{g})^{c}$ by $I$. Regarding $T^{c}$ as a subset of $\mathcal{K}$ leaves its singularity-type unchanged. By transversality, and since $\mathcal{E}=X_{H}^{-1}\left(\mathcal{T}^{c}\right)$, this is also the singularity type of $\mathcal{E}$ at $p_{e}$. Since $G$ acts freely on $P$ the set $\mathcal{E}$ is locally isomorphic to the product of $\mathcal{E} / G$ and $G$ and so $\mathcal{E} / G$ has the same singularity type at $\bar{p}_{e}$ as $\mathcal{E}$ does at $p_{e}$.

We end this section with a result that shows that all the relative equilibria of 'most' $G$-invariant Hamiltonians are transversal. Recall that $G$ is a connected, compact Lie group acting freely and symplectically on the symplectic manifold $(P, \omega)$. Let $C_{G}^{\infty}(P)$ denote the set of all $G$-invariant $C^{\infty}$ functions on $P$ and $C^{\infty}(P / G)$ the set of all $C^{\infty}$ functions on the orbit space $P / G$. Pulling back functions by the orbit map $\pi: P \rightarrow P / G$ defines a bijection $\pi^{*}: C^{\infty}(P / G) \cong C_{G}^{\infty}(P / G)$. We give $C^{\infty}(P / G)$ the Whitney $C^{\infty}$ topology and $C_{G}^{\infty}(P)$ the isomorphic topology induced by $\pi^{*}$.

Theorem 3 There exists a dense subset $\mathcal{H} \subset C_{G}^{\infty}(P)$ such that if $H \in \mathcal{H}$ then every relative equilibrium of $H$ is transversal.

Proof For any $G$-invariant function $H$ on $P$ let $\bar{H}$ denote the quotient function on $P / G$. The derivative $d H: P \rightarrow \mathcal{T}^{o} \subset T^{*} P$ is $G$-equivariant and so descends to a mapping from $P / G$ to $\mathcal{T}^{o} / G$ which we will denote by $(d H)^{-}$. Since $G$ is acting freely on $P$ the orbit space $P / G$ is a smooth manifold and $\mathcal{T}^{\circ} / G$ is a smooth vector bundle over $P / G$ which can be identified with the cotangent bundle $T^{*}(P / G)$. Under this identification $(d H)^{-}$becomes equal to $d \bar{H}$.

The Hamiltonian $H$ has a relative equilibrium at $p_{e} \in P$ if and only if $d H\left(p_{e}\right) \in \mathcal{K}^{o c}$ and so if and only if $d \bar{H}\left(\bar{p}_{e}\right) \in \mathcal{K}^{o c} / G$. The set $\left\{\mathcal{K}_{(K)}^{o c} / G\right.$ : $\left.(K) \in \mathcal{I}^{c}\right\}$ is a Whitney regular stratification of $\mathcal{K}^{o c} / G \subset T^{*}(P / G)$ and $p_{e}$ is a transversal relative equilibrium if and only if $d \bar{H}: P / G \rightarrow T^{*}(P / G)$ is transversal to this stratification at $\bar{p}_{e}$. It now follows from the Thom transversality theorem that the set of functions $\bar{H}$ on $P / G$ for which the derivatives $d \bar{H}$ are everywhere transversal to this stratification is dense in $C^{\infty}(P / G)$. Pulling this back to $C_{G}^{\infty}(P)$ gives the required set $\mathcal{H}$.

Thus for $H$ in the set $\mathcal{H}$ given by the Theorem the set of all relative equilibria $\mathcal{E} \subset P$ is Whitney stratified by the types of the relative equilibria, the manifold 
$\mathcal{E}_{(K)}$ of relative equilibria of type $(K)$ has dimension $\operatorname{dim} G+2 \operatorname{dim} Z(K)-\operatorname{dim} K$, and the singularity-type of $\mathcal{E}$ at a point in $\mathcal{E}_{(K)}$ is $\left(\mathfrak{l}^{*} \oplus \mathfrak{l}\right)^{c}$ where $\mathfrak{l}$ is the Lie algebra of $K / Z(K)$.

\section{Linearization of a Transversal Relative Equi- librium}

In this section we give an alternative form of the transversality condition using a normal form for the linearization of a vector field at a relative equilibrium due to Patrick [1998]. This is then used to describe the tangent spaces of the strata of $\mathcal{E} / G$ and to prove a generalization and partial converse of a result of Patrick 1998 on the symplectic properties of these strata.

Recall that $\mathcal{T}_{p_{e}}=\mathfrak{g} \cdot p_{e}$ and $\mathcal{K}_{p_{e}}=\operatorname{ker} d J\left(p_{e}\right)$, and decompose $T_{p_{e}} P$ as a direct sum

$$
T_{p_{e}} P=T_{0} \oplus N_{1} \oplus N_{0} \oplus T_{1}
$$

as follows. Let $T_{0}=\mathfrak{g}_{\mu_{e}} \cdot p_{e}$. Let $\mathfrak{g}_{\mu_{e}}^{\perp}$ be a $G$-invariant complement to $\mathfrak{g}_{\mu_{e}}$ in $\mathfrak{g}$ and set $T_{1}=\mathfrak{g}_{\mu_{e}}^{\perp} \cdot p_{e}$. Choose a complement $N_{1}$ to $T_{0}$ within $\mathcal{K}_{p_{e}}$ and a complement $N_{0}$ to $\mathcal{T}_{p_{e}} \oplus \mathcal{K}_{p_{e}}$ within $T_{p_{e}} P$. The subspace $N_{1}$ is a symplectic normal space at $p_{e}$ and can be identified with the tangent space to the reduced phase space $P_{\mu_{e}}$ at $\bar{p}_{e}=G . p_{e}$. The subspace $N_{0}$ can be identified with $\mathfrak{g}_{\mu_{e}}^{*}$, regarded as a subspace of $\mathfrak{g}^{*}$, via the map $d J\left(p_{e}\right) \mid N_{0}$. Since $p_{e}$ is regular, we have $T_{0} \cong \mathfrak{g}_{\mu_{e}}$ and $T_{1} \cong \mathfrak{g}_{\mu_{e}}^{\perp}$, and so (1) becomes

$$
T_{p_{e}} P \cong \mathfrak{g}_{\mu_{e}} \oplus T_{\bar{p}_{e}} P_{\mu_{e}} \oplus \mathfrak{g}_{\mu_{e}}^{*} \oplus \mathfrak{g}_{\mu_{e}}^{\perp} .
$$

The symplectic form with respect to this decomposition is the product of the reduced form on $T_{\bar{p}_{e}} P_{\mu_{e}}$, the canonical form on $\mathfrak{g}_{\mu_{e}} \oplus \mathfrak{g}_{\mu_{e}}^{*}$, and the KostantSouriau form on $\mathfrak{g}_{\mu_{e}}^{\perp}=T_{\mu_{e}}\left(G . \mu_{e}\right)$. The map $d J\left(p_{e}\right)$ has the explicit form

$$
d J\left(p_{e}\right)\left(\xi_{0} \oplus w \oplus \mu_{0} \oplus \xi_{1}\right)=\mu_{0}+\operatorname{coad}_{\xi_{1}} \mu_{e} .
$$

A relative equilibrium $p_{e}$ of $H$ is an equilibrium point of $X_{H_{\xi_{e}}}$ where $H_{\xi_{e}}=$ $H-J_{\xi_{e}}$. Patrick 1998] shows that the complements $N_{0}$ and $N_{1}$ can be chosen so that, with respect to the decomposition (2), the linearization of $X_{H_{\xi_{e}}}$ at $p_{e}$ has the form

$$
d X_{H_{\xi_{e}}}\left(p_{e}\right)=\left[\begin{array}{cccc}
-\operatorname{ad}_{\xi_{e}} \mid \mathfrak{g}_{\mu_{e}} & C^{*} & D & 0 \\
0 & d X_{H_{\mu_{e}}}\left(\bar{p}_{e}\right) & C & 0 \\
0 & 0 & -\operatorname{coad}_{\xi_{e}} \mathfrak{g}_{\mu_{e}}^{*} & 0 \\
0 & 0 & 0 & -\operatorname{ad}_{\xi_{e}} \mid \mathfrak{g}_{\mu_{e}}^{\perp}
\end{array}\right]
$$

where $H_{\mu_{e}}$ is the induced Hamiltonian on the reduced phase space $P_{\mu_{e}}, X_{H_{\mu_{e}}}$ is the associated Hamiltonian vector field and $d X_{H_{\mu_{e}}}\left(\bar{p}_{e}\right)$ is its linearization at the equilibrium point $\bar{p}_{e}$. The operator $D: \mathfrak{g}_{\mu_{e}}^{*} \cong N_{0} \rightarrow T_{0} \cong \mathfrak{g}_{\mu_{e}}$ describes the drift 
along the group orbit and $C: \mathfrak{g}_{\mu_{e}}^{*} \cong N_{0} \rightarrow N_{1}$ describes interactions between the reduced dynamics and the motion along group orbits due to possible 1-1 resonances between these two motions. If the spectrum of $\operatorname{ad}_{\xi_{e}}$ is distinct from the spectrum of $d X_{H_{\mu_{e}}}\left(\bar{p}_{e}\right)$ then such interactions do not occur to first order and $N_{0}$ and $N_{1}$ can be chosen so that $C=0$. In general the dual operator $C^{*}$ is regarded as a map from $N_{1}$ (instead of $N_{1}^{*}$ ) by using the symplectic form to identify $N_{1}$ with its dual. The operators $C, C^{*}$ and $D$ enjoy the following properties:

$$
\begin{gathered}
d X_{H_{\mu_{e}}}\left(\bar{p}_{e}\right) C=-C \operatorname{coad}_{\xi_{e}}, \\
C^{*} d X_{H_{\mu_{e}}}\left(\bar{p}_{e}\right)=-\operatorname{ad}_{\xi_{e}} C^{*}, \\
\operatorname{ad}_{\xi_{e}} D=D \operatorname{coad}_{\xi_{e}},
\end{gathered}
$$

and the operator $D$ is symmetric.

As in the theorems of the previous section we will set $K_{e}=G_{\mu_{e}} \cap G_{\xi_{e}}$, denote by $\mathfrak{k}_{e}$ the Lie algebra of $K_{e}, \mathfrak{z}_{e}$ the center of $\mathfrak{k}_{e}$, and $\mathfrak{l}_{e}=\mathfrak{k}_{e} / \mathfrak{z}_{e}$. As a consequence of (5), $C$ maps $\mathfrak{k}_{e}^{*}$ into ker $d X_{H_{\mu_{e}}}\left(\bar{p}_{e}\right)$, since $\operatorname{coad}_{\xi_{e}}$ is zero on $\mathfrak{k}_{e}^{*}$. Equation (6) similarly implies that $C^{*}$ maps ker $d X_{H_{\mu_{e}}}\left(\bar{p}_{e}\right)$ into $\mathfrak{k}_{e}$ while equation (7) implies that $D$ maps $\mathfrak{k}_{e}^{*}$ into $\mathfrak{k}_{e}$. The next theorem characterizes transversal relative equilibria in terms of the above normal form for $d X_{H_{\xi_{e}}}\left(p_{e}\right)$. Recall that $p_{e}$ is said to be nondegenerate if $d X_{H_{\mu_{e}}}\left(\bar{p}_{e}\right)$ is invertible.

Theorem 4 The relative equilibrium $p_{e}$ is transversal if and only if all the following three conditions hold:

1. either $p_{e}$ is nondegenerate or 0 is a semisimple eigenvalue of $d X_{H_{\mu_{e}}}\left(\bar{p}_{e}\right)$;

2. $C$ maps $\mathfrak{z}_{e}^{*} \subset \mathfrak{k}_{e}^{*} \subset \mathfrak{g}_{\mu_{e}}^{*}$ onto $\operatorname{ker} d X_{H_{\mu_{e}}}\left(\bar{p}_{e}\right)$;

3. $C^{*}\left(\operatorname{ker} d X_{H_{\mu_{e}}}\left(\bar{p}_{e}\right)\right)+D\left(\operatorname{ker} C \cap \mathfrak{z}_{e}^{*}\right)+\mathfrak{z}_{e}=\mathfrak{k}_{e}$.

Proof From Patrick 1995, page 409, there is the following formula for the derivative of $\psi: P \times \mathfrak{g} \rightarrow T P$ at $\left(p_{e}, \xi_{e}\right)$

$$
T_{\left(p_{e}, \xi_{e}\right)} \psi(v, \eta)=\left(v, d X_{H_{\xi_{e}}}\left(p_{e}\right) v-\eta \cdot p_{e}\right),
$$

where on the right side $T_{0_{p_{e}}}(T P)$ has been decomposed into horizontal and vertical parts. By Lemma 3, $p_{e}$ is transversal if and only if $\psi_{\left(K_{e}\right)}:(P \times \mathfrak{g})_{\left(K_{e}\right)}^{c} \rightarrow$ $\mathcal{K}$ is transversal to the zero section of $\mathcal{K}$ at $\left(p_{e}, \xi_{e}\right)$, which is equivalent to

$$
\operatorname{ker} d J\left(p_{e}\right) \subseteq\left\{d X_{H_{\xi_{e}}}\left(p_{e}\right) v-\eta \cdot p_{e}:(v, \eta) \in T_{\left(p_{e}, \xi_{e}\right)}(P \times \mathfrak{g})_{\left(K_{e}\right)}^{c}\right\},
$$

while from Propositions 1 and 2 .

$$
\begin{aligned}
& T_{\left(p_{e}, \xi_{e}\right)}(P \times \mathfrak{g})_{\left(K_{e}\right)}^{c} \\
& \quad=\left\{(v, \eta) \in T_{p_{e}} P \times \mathfrak{g}:\left(d J\left(p_{e}\right) v, \eta\right) \in T_{\left(\mu_{e}, \xi_{e}\right)}\left(\mathfrak{g}^{*} \oplus \mathfrak{g}\right)_{\left(K_{e}\right)}^{c}\right\}
\end{aligned}
$$




$$
=\left\{(v, \eta) \in T_{p_{e}} P \times \mathfrak{g}:\left(d J\left(p_{e}\right) v, \eta\right) \in\left(\mathfrak{z}_{e}^{*} \oplus \mathfrak{z}_{e}\right) \oplus \mathfrak{g} \cdot\left(\mu_{e}, \xi_{e}\right)\right\} .
$$

Using (3) and (4) transversality is equivalent to

$$
\begin{aligned}
\mathfrak{g}_{\mu_{e}} \oplus N_{1} & =\left\{\left(-\operatorname{ad}_{\xi_{e}} \xi_{0}+C^{*} w+D \mu_{0}-\eta_{0}, d X_{H_{\mu_{e}}}\left(\bar{p}_{e}\right) w+C \mu_{0}\right):\right. \\
\xi_{0}, \eta_{0} & \left.\in \mathfrak{g}_{\mu_{e}}, w \in T_{\bar{p}_{e}} P_{\mu_{e}}, \mu_{0} \in \mathfrak{g}_{\mu_{e}}^{*} \text { and conditions (10)-(13) hold }\right\},
\end{aligned}
$$

the conditions (10)-(13) being that there exist $\xi_{1}, \eta_{1} \in \mathfrak{g}_{\mu_{e}}^{\perp}, \zeta \in \mathfrak{z}_{e}^{*}, z \in \mathfrak{z}_{e}$ and $\tilde{\xi} \in \mathfrak{g}$ such that

$$
\begin{gathered}
\operatorname{coad}_{\xi_{e}} \mu_{0}=0, \\
\eta_{1}=-\operatorname{ad}_{\xi_{e}} \xi_{1}, \\
\mu_{0}+\operatorname{coad}_{\xi_{1}} \mu_{e}=\zeta+\operatorname{coad}_{\tilde{\xi}} \mu_{e}, \\
\eta_{0}+\eta_{1}=z+\operatorname{ad}_{\tilde{\xi}} \xi_{e} .
\end{gathered}
$$

Condition (10) gives $\mu_{0} \in \operatorname{ann}\left(\mathfrak{g}_{\mu_{e}} \cdot \xi_{e}\right)=\mathfrak{k}_{e}^{*}$. Inserting (11) into (13), one sees that (10)-(13) are equivalent to the conditions that $\mu_{0} \in \mathfrak{k}_{e}^{*}$ and there exist $\zeta \in \mathfrak{z}_{e}^{*}, z \in \mathfrak{z}_{e}$ and $\tilde{\xi} \in \mathfrak{g}$ such that

$$
\begin{gathered}
\mu_{0}=\zeta+\operatorname{coad}_{\tilde{\xi}-\xi_{1}} \mu_{e}, \\
\eta_{0}=z+\operatorname{ad}_{\tilde{\xi}-\xi_{1}} \xi_{e} .
\end{gathered}
$$

In (14) $\mu_{0}$ and $\zeta$ lie in $\mathfrak{k}_{e}^{*}$ which is orthogonal to the image of coad and so (14) is equivalent to $\mu_{0} \in \mathfrak{z}_{e}^{*}$ and $\tilde{\xi}-\xi_{1} \in \mathfrak{g}_{\mu_{e}}$. Since the right side of (15) is then clearly in $\mathfrak{g}_{\mu_{e}}$, after substitution of (15) into (9), transversality becomes equivalent to

$$
\begin{array}{r}
\mathfrak{g}_{\mu_{e}} \oplus N_{1}=\left\{\left(C^{*} w+D \mu_{0}+g_{\mu_{e}} \cdot \xi_{e}+\mathfrak{z}_{e}, d X_{H_{\mu_{e}}}\left(\bar{p}_{e}\right) w+C \mu_{0}\right):\right. \\
\left.w \in T_{\bar{p}_{e}} P_{\mu_{e}}, \mu_{0} \in \mathfrak{z}_{e}^{*}\right\} .
\end{array}
$$

Let $E_{0}$ be the generalized eigenspace of $d X_{H_{\mu_{e}}}\left(\bar{p}_{e}\right)$ associated to the eigenvalue 0 and let $E_{1}$ be the sum of the other generalized eigenspaces, so that $N_{1}=E_{0} \oplus E_{1}$. This decomposition is preserved by $d X_{H_{\mu_{e}}}\left(\bar{p}_{e}\right)$ and for $j=0,1$ we denote the restriction of $d X_{H_{\mu_{e}}}\left(\bar{p}_{e}\right)$ to $E_{j}$ by $L_{j}$. It follows from (16) that for $p_{e}$ to be transversal we must have image $L_{0}+$ image $C \supseteq E_{0}$. Since image $C \subset \operatorname{ker} d X_{H_{\mu_{e}}}\left(\bar{p}_{e}\right)=\operatorname{ker} L_{0} \subset E_{0}$ this implies image $L_{0}+\operatorname{ker} L_{0}=E_{0}$. For a nilpotent operator, such as $L_{0}$, an application of the Jordan normal form shows that this is only possible if $L_{0}=0$. It follows that if $p_{e}$ is transversal then either it is nondegenerate or 0 is a semisimple eigenvalue of $d X_{H_{\mu_{e}}}\left(\bar{p}_{e}\right)$.

So in the proving of either direction of the theorem we may assume $L_{0}=0$. But then the transversality condition (16) reduces to

$$
\begin{array}{r}
\mathfrak{k}_{e} \oplus \operatorname{ker} d X_{H_{\mu_{e}}}\left(\bar{p}_{e}\right)=\left\{\left(C^{*} w+D \mu_{0}+\mathfrak{g}_{\mu_{e}} \cdot \xi_{e}+\mathfrak{z}_{e}, C \mu_{0}\right):\right. \\
\left.w \in \operatorname{ker} d X_{H_{\mu_{e}}}\left(\bar{p}_{e}\right), \mu_{0} \in \mathfrak{z}_{e}^{*}\right\} .
\end{array}
$$

As $C^{*}$ maps ker $d X_{H_{\mu_{e}}}\left(\bar{p}_{e}\right)$ into $\mathfrak{k}_{e}$, while $D$ maps $\mathfrak{z}_{e}^{*}$ into $\mathfrak{k}_{e}, \mathfrak{z}_{e} \subseteq \mathfrak{k}_{e}$, and $\mathfrak{g}_{\mu_{e}} \cdot \xi_{e}=\mathfrak{k}_{e}^{\perp} \cap \mathfrak{g}_{\mu_{e}},(17)$ is equivalent to the pair of statements 2 and 3 in the Theorem. 
Let $\bar{D}: \mathfrak{z}_{e}^{*} \rightarrow \mathfrak{l}_{e} \cong \mathfrak{k}_{e} / \mathfrak{z}_{e}$ denote the mapping obtained by the restricting the drift operator $D$ to $\mathfrak{z}_{e}^{*}$ and then composing with the projection from $\mathfrak{k}_{e}$ to $\mathfrak{l}_{e} \cong \mathfrak{k}_{e} / \mathfrak{z}_{e}$.

\section{Corollary 3}

1. If $p_{e}$ is nondegenerate then $p_{e}$ is transversal if and only if $\bar{D}$ is surjective.

2. If $p_{e}$ is transversal then

$$
\operatorname{dim} \operatorname{ker} d X_{H_{\mu_{e}}}\left(\bar{p}_{e}\right) \leq 2 \operatorname{dim} Z\left(K_{e}\right)-\operatorname{dim} K_{e} .
$$

Proof If $p_{e}$ is nondegenerate then $\operatorname{ker} d X_{H_{\mu_{e}}}\left(\bar{p}_{e}\right)=\{0\}, \operatorname{ker} C=\mathfrak{g}_{\mu_{e}}^{*}$ and the transversality conditions reduce to $D\left(\mathfrak{z}_{e}^{*}\right)+\mathfrak{z}_{e}=\mathfrak{k}_{e}$, as required.

The second statement follows immediately from the third transversality condition in Theorem 1 .

We note that if $K_{e}$ is a maximal torus then $\mathfrak{z}_{e}=\mathfrak{k}_{e}$ and so $\bar{D}$ is trivially surjective and nondegeneracy implies transversality. However in general a nontrivial nondegeneracy condition must be satisfied by the drift operator for a relative equilibrium to be transversal.

The normal form (位) also enables us to give a description of the tangent spaces to the manifolds $\mathcal{E}_{\left(K_{e}\right)}$ at transversal relative equilibria.

Theorem 5 If the relative equilibrium $p_{e}$ is transversal then, with respect to the decomposition (国),

$$
\begin{array}{r}
T_{p_{e}} \mathcal{E}_{\left(K_{e}\right)}=\left\{\xi_{0} \oplus w \oplus \mu_{0} \oplus \xi_{1}: \xi_{0} \in \mathfrak{g}_{\mu_{e}}, \xi_{1} \in \mathfrak{g}_{\mu_{e}}^{\perp}, \mu_{0} \in \mathfrak{z}_{e}^{*} \cap \operatorname{ker} C,\right. \\
\left.w \in \operatorname{ker} d X_{H_{\mu_{e}}}\left(\bar{p}_{e}\right), C^{*} w+D \mu_{0} \in \mathfrak{z}_{e}\right\} .
\end{array}
$$

Proof Since $\mathcal{E}_{\left(K_{e}\right)}$ is the projection to $P$ of $\psi_{\left(K_{e}\right)}^{-1}(Z(T P)) \subset(P \times \mathfrak{g})_{\left(K_{e}\right)}^{c}$, and in view of (8), we have

$$
\begin{aligned}
T_{p_{e}} \mathcal{E}_{\left(K_{e}\right)}=\left\{v \in T_{p_{e}} P: d X_{H_{\xi_{e}}}\left(p_{e}\right) v-\eta \cdot p_{e}=0\right. \\
\left.\quad \text { for some } \eta \in \mathfrak{g} \text { such that }(v, \eta) \in T_{\left(p_{e}, \xi_{e}\right)}(P \times \mathfrak{g})_{\left(K_{e}\right)}^{c}\right\},
\end{aligned}
$$

which, in terms of the local normal form (价, becomes

$$
T_{p_{e}} \mathcal{E}_{\left(K_{e}\right)}=\left\{\xi_{0} \oplus w \oplus \mu_{0} \oplus \xi_{1}: \text { the conditions directly below hold }\right\},
$$

the conditions being that there exists $\eta_{0} \in \mathfrak{g}_{\mu_{e}}$ and $\eta_{1} \in \mathfrak{g}_{\mu_{e}}^{\perp}$ such that

$$
\begin{gathered}
-\operatorname{ad}_{\xi_{e}} \xi_{0}+C^{*} w+D \mu_{0}-\eta_{0}=0, \quad d X_{H_{\mu_{e}}}\left(\bar{p}_{e}\right) w+C \mu_{0}=0 \\
\operatorname{coad}_{\xi_{e}} \mu_{0}=0, \quad-\operatorname{ad}_{\xi_{e}} \xi_{1}-\eta_{1}=0
\end{gathered}
$$


subject to the constraints that there exist $\zeta \in \mathfrak{z}_{e}^{*}, z \in \mathfrak{k}_{e}$ and $\tilde{\xi} \in \mathfrak{g}$ such that

$$
\mu_{0}+\operatorname{coad}_{\xi_{1}} \mu_{e}=\zeta+\operatorname{coad}_{\tilde{\xi}} \mu_{e}, \quad \eta_{0}+\eta_{1}=z+\operatorname{ad}_{\tilde{\xi}} \xi_{e} .
$$

As in the proof of Theorem (4), these conditions are equivalent to the conditions that there exist $\tilde{\xi} \in \mathfrak{g}$ and $z \in \mathfrak{z} e$ such that

$$
\begin{gathered}
\mu_{0} \in \mathfrak{z}_{e}, \quad C \mu_{0}=0, \quad d X_{H_{\mu_{e}}}\left(\bar{p}_{e}\right) w=0 \\
-\operatorname{ad}_{\xi_{e}} \xi_{0}+C^{*} w+D \mu_{0}-z-\operatorname{ad}_{\tilde{\xi}-\xi_{1}} \xi_{e}=0
\end{gathered}
$$

subject only to the constraint that $\tilde{\xi}-\xi_{1} \in \mathfrak{g}_{\mu_{e}}$, as long as one takes

$$
\eta_{1}=-\operatorname{ad}_{\xi_{e}} \xi_{0}, \quad \eta_{0}=z+\operatorname{ad}_{\tilde{\xi}-\xi_{1}} \xi_{e} .
$$

Again as in the proof of Theorem (4), (18) and $(19)$ are equivalent to $(18)$ and

$$
C^{*} w+D \mu_{0}-z=0, \quad \operatorname{ad}_{\tilde{\xi}-\xi_{1}-\xi_{0}} \xi_{e}=0,
$$

or equivalently (18), $C^{*} w+D \mu_{0} \in \mathfrak{z} e$ and $\tilde{\xi}-\xi_{1}-\xi_{0} \underset{\tilde{\xi}}{\in \mathfrak{k}_{e}}$. This proves the theorem since for any $\xi_{0}$ and $\xi_{1}$ we can find $\tilde{\xi}$ such that $\tilde{\xi}-\xi_{1}-\xi_{0} \in \mathfrak{k}_{e}$.

One can calculate the linearized dependence at $p_{e}$ of the generators of the relative equilibria on the relative equilibria themselves by taking, at the end of the proof of Theorem $\mathrm{f}, \tilde{\xi}-\xi_{1}-\xi_{0}=k \in \mathfrak{k}$, and substituting this and $z$ from (21) into (20), which gives

$$
\eta_{0}=C^{*} w+D \mu_{0}-\operatorname{ad}_{\xi_{e}} \xi_{0}, \quad \eta_{1}=-\operatorname{ad}_{\xi_{e}} \xi_{1} .
$$

If we define

$$
\hat{\mathcal{E}}_{\left(K_{e}\right)}=\psi_{\left(K_{e}\right)}^{-1}(Z(T P)),
$$

that is $\hat{\mathcal{E}}_{\left(K_{e}\right)}$ is the set of relative equilibria paired with their generators, then

$$
T_{p_{e}} \hat{\mathcal{E}}_{\left(K_{e}\right)}=\left\{(v, \eta) \in T_{p_{e}} P \times \mathfrak{g}: d X_{H_{\xi_{e}}}\left(p_{e}\right) v-\eta \cdot p_{e}=0\right\},
$$

and so $T_{p_{e}} \hat{\mathcal{E}}_{\left(K_{e}\right)}$ is the graph of (22) over the tangent space $T_{p_{e}} \mathcal{E}_{\left(K_{e}\right)}$ calculated in Theorem 5 .

The following result uses Theorem 5 to give a generalization and partial converse of a result of Patrick 1995.

Theorem 6 If the relative equilibrium $p_{e}$ is transversal then $\mathcal{E}_{\left(K_{e}\right)}$ is a symplectic submanifold of $P$ in a neighborhood of $p_{e}$ if and only if $p_{e}$ is nondegenerate and $G_{\mu_{e}}$ is a maximal torus of $G$. 
Proof The manifold $\mathcal{E}_{\left(K_{e}\right)}$ is symplectic near $p_{e}$ if and only if the tangent space $T_{p_{e}} \mathcal{E}_{\left(K_{e}\right)}$ is a symplectic subspace of $T_{p_{e}} P$. From Theorem 5 and the description of the symplectic form on $T_{p_{e}} P$ in terms of the decomposition (2) it is clear that the restriction of the symplectic form to $T_{p_{e}} \mathcal{E}_{\left(K_{e}\right)}$ is degenerate on the subspace $\operatorname{ann}_{\mathfrak{g}_{\mu_{e}}}\left(\mathfrak{z}_{e}^{*}\right) \subset \mathfrak{g}_{\mu_{e}} \cong T_{0}$. Thus $T_{p_{e}} \mathcal{E}_{\left(K_{e}\right)}$ can only be a symplectic subspace of $T_{p_{e}} P$ if $\operatorname{ann}_{\mathfrak{g}_{\mu_{e}}}\left(\mathfrak{z}_{e}^{*}\right)=\{0\}$, that is $\mathfrak{g}_{\mu_{e}}=\mathfrak{z}_{e}$. This implies that $\mathfrak{g}_{\mu_{e}}=\mathfrak{k}_{e}$ and is abelian, and so $G_{\mu_{e}}$ is a maximal torus of $G$ by Lemma 1 .

When $\mathfrak{z}_{e}=\mathfrak{k}_{e}=\mathfrak{g}_{\mu_{e}}$ the condition $C^{*} w+D \mu_{0} \in \mathfrak{z}_{e}$ in the description of $T_{p_{e}} \mathcal{E}_{\left(K_{e}\right)}$ in Theorem 5 is automatically satisfied. With respect to the decomposition (2) we therefore have

$$
T_{p_{e}} \mathcal{E}_{\left(K_{e}\right)}=\mathfrak{g}_{\mu_{e}} \oplus \operatorname{ker} d X_{H_{\mu_{e}}}\left(\bar{p}_{e}\right) \oplus \operatorname{ker} C \oplus \mathfrak{g}_{\mu_{e}}^{\perp} .
$$

The subspace $\mathfrak{g}_{\mu_{e}}^{\perp}$ is always symplectic. Since $p_{e}$ is transversal any 0 eigenvalue of $d X_{H_{\mu_{e}}}\left(\bar{p}_{e}\right)$ is semisimple and so $\operatorname{ker} d X_{H_{\mu_{e}}}\left(\bar{p}_{e}\right)$ is symplectic. It follows that $T_{p_{e}} \mathcal{E}_{\left(K_{e}\right)}$ is a symplectic subspace of $T_{p_{e}} P$ if and only if $\operatorname{ker} C=\mathfrak{g}_{\mu_{e}}^{*}$ and so $C=0$. However, for a transversal relative equilibrium $C$ must map onto ker $d X_{H_{\mu_{e}}}\left(\bar{p}_{e}\right)$ and so $T_{p_{e}} \mathcal{E}_{\left(K_{e}\right)}$ can only be a symplectic subspace of $T_{p_{e}} P$ if $p_{e}$ is nondegenerate.

Conversely, suppose $p_{e}$ is nondegenerate and $G_{\mu_{e}}$ is a maximal torus. Then $K_{e} \subseteq G_{\mu_{e}}, K_{e}$ contains a maximal torus and so $K=G_{\mu_{e}}$ and $\mathfrak{z}_{e}=\mathfrak{k}_{e}=\mathfrak{g}_{\mu_{e}}$. It follows that $C=0$, equation (23) holds, and $T_{p_{e}} \mathcal{E}_{\left(K_{e}\right)}$ is a symplectic subspace of $T_{p_{e}} P$.

\section{Acknowledgments}

The authors are grateful to the UK EPSRC for a Visiting Fellowship grant. G. W. Patrick was partially supported by NSERC grant OGP010571.

\section{References}

Adams, J. F. [1969]. Lectures on Lie Groups. University of Chicago Press.

Arms, J. M., J. E. Marsden, and V. Moncrief [1981]. Bifurcations of momentum mappings. Comm. Math. Phys. 78, 455-478.

Goresky, M. and R. MacPherson [1998]. Stratified Morse Theory. Springer-Verlag.

Lerman, E. and S. F. Singer [1998]. Relative equilibria at singular points of the momentum map. Nonlinearity 11, 1637-1649.

Montaldi, J. [1997]. Persistence and stability of relative equilibria. Nonlinearity 10, 449466.

Ortega, J.-P. and T. S. Ratiu [1997]. Persistence and smoothness of critical relative elements in Hamiltonian systems with symmetry. C. R. Acad. Sci. Paris Sér. I Math. 325, 11071111.

Patrick, G. W. [1995]. Relative equilibria of Hamiltonian systems with symmetry: linearization, smoothness, and drift. J. Nonlin. Sc. 5, 373-418.

Patrick, G. W. [1998]. Dynamics near relative equilibria: Nongeneric momenta at a 1:1 group-reduced resonance. Submitted Math. Z. 
Roberts, R. M. and M. E. R. de Sousa Dias [1997]. Bifurcations from relative equilibria of Hamiltonian systems. Nonlinearity 10, 1719-1738.

Sjamaar, R. and E. Lerman [1991]. Stratified symplectic spaces and reduction. Ann. of Math. 134, 375-422. 